\title{
Malignant Frontal Sinus Neoplasm
}

National Cancer Institute

\section{Source}

National Cancer Institute. Malignant Frontal Sinus Neoplasm. NCI Thesaurus. Code C3542.

A primary or metastatic malignant neoplasm involving the frontal sinus. 